\title{
A Novel Approach to Scatter-free Imaging for the Improvement of Breast Cancer Detection
}

\author{
F.H.Green ${ }^{a}$, M. C. Veale ${ }^{b}$, M. D. Wilson ${ }^{b}$, P. Seller $^{\mathrm{b}}$, J. Scuffham $^{\mathrm{c}}$, S.Pani $^{\mathrm{a}}$ \\ ${ }^{a}$ Department of Physics, University of Surrey, Guildford, UK \\ ${ }^{b}$ STFC Rutherford Appleton Laboratories, Didcot, UK \\ ${ }^{c}$ Department of Nuclear Medicine, Royal Surrey County Hospital, Guildford, UK \\ E-mail: f.h.green@surrey.ac.uk
}

\begin{abstract}
Compton scattering is one of the main causes of image degradation in X-ray imaging. This is particularly noticeable in mammography where details of interest feature low contrast in comparison to the surrounding tissue. This work shows the feasibility of obtaining scatter-free images by using a quasimonochromatic X-ray beam and a pixellated spectroscopic detector. This work presents characterisation of the imaging system and quantitative imaging data of a low contrast test object. An improvement in contrast by $8 \%$ was observed compared to images obtained including scattered radiation. Comparison with a conventional setup showed an increase in the image quality factor when scatter has been removed.
\end{abstract}

KEYWORDS: X-ray mammography; X-ray monochromators; Pixellated detectors. 


\section{Contents}

2. Materials and methods

2.1 Experimental setup

2.2 Image acquisition and processing

2.3 Contrast and signal to noise ratio (SNR)

\section{Results}

3.1 Crystal characterisation

3.2 Imaging of low energy test object

\section{Conclusions}

\section{Introduction}

One of the causes of image degradation in X-ray imaging is Compton scattering of X-rays. Scatter reduces the contrast of the image and therefore the overall image quality [1].

Mammography is an imaging modality that presents particular challenges as structures that suggest the presence of breast cancer either have low contrast (nodules) or small size (calcifications) [2],[3]. Breast tissue is also very sensitive to radiation and so the dose needs to be kept as low as possible so as to limit the risk of radiation-induced carcinogenesis [4].

Current radiographic techniques use an anti-scatter grid in order to reduce the quantity of scattered x-rays from reaching the imaging plate and degrading the image. This method has its drawbacks because as well as absorbing scattered X-rays, a proportion of the primary beam will also be absorbed. To counteract this loss in intensity on the detector, the dose to the patient is increased [5].

This project investigates an alternative, unique method for scatter removal in mammography using monochromatic X-rays from a conventional X-ray source combined with a pixellated spectroscopic detector.

The combination of a monochromator with a detector that has spectroscopic capabilities allows the windowing out of scattered X-rays. Compton-scattered X-rays loose energy in the scattering process and 
will therefore have a lower energy than the primary beam and can be removed by simply windowing the spectrum around the primary beam peak.

Another benefit to using this setup is energy tuneability; the energy can be tuned to suit the breast thickness of the individual. This has been shown to work for both synchrotron [6] and conventional sources [7].

An additional advantage to using a monochromatic beam is it removes lower energy X-rays that increase the dose when fully absorbed by the breast. Higher energy X-rays that are more likely to scatter and pass through without contributing to the image are also removed. This improves image quality and reduces dose.

HEXITEC (High Energy X-ray Imaging Technology) is a collaborative project between Manchester, Durham, Surrey Universities, Birkbeck College, the Science Techonology Facilities Council (STFC) and it involves collaboration with the Royal Surrey County Hospital and the University College London and aims to develop spectroscopic, photon counting detectors for the use with high-energy X-ray and $\gamma$-ray spectroscopy. A pixellated spectroscopic detector combines spectral information with the spatial information of a conventional imaging detector. The HEXITEC pixellated spectroscopic detector used in these studies has been used in the development of several fields including radiation detection in space science [8] and illegal substance/weapons detection [9]. It also has several medical applications in both nuclear medicine [10] and X-ray imaging [11].

\section{Materials and Methods}

\subsection{Experimental Setup}

The experimental setup for the study is shown in figure 1 and consists of a Hamamatsu L8231-01 X-ray source with a tungsten anode operated at $50 \mathrm{kV}$ (for present work), a $1 \mathrm{~cm}$ thick brass collimator with a 1 $\mathrm{mm} \times 20 \mathrm{~mm}$ slit, a monochromator, the test object to be imaged and a HEXITEC pixellated spectroscopic detector. 


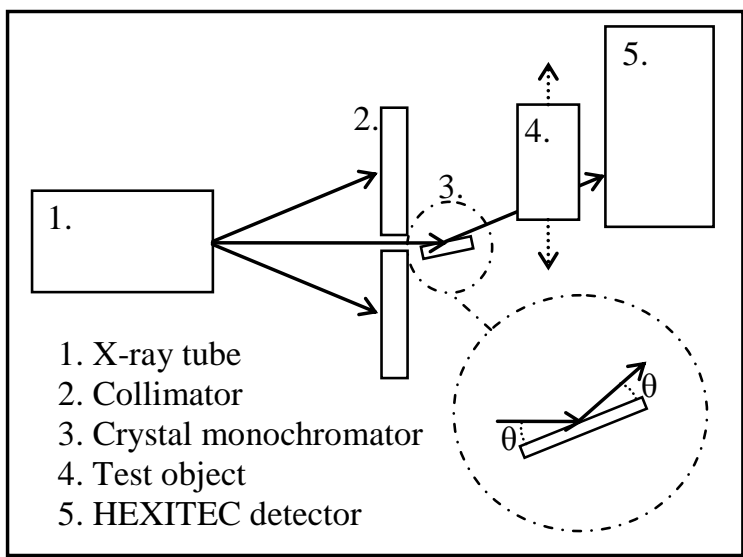

Figure 1, a schematic of the experimental setup where $\theta$ is the diffraction angle.

\section{a. Monochromator}

The monochromator is made from a $20 \times 20 \times 2 \mathrm{~mm}$ Highly Oriented Pyrolytic Graphite (HOPG) crystal with a mosaic spread of $0.4 \pm 0.1$ degrees [12]. The crystal is mounted on a goniometer so that the incident angle of the X-ray beam on the crystal can be varied in accordance with Bragg's condition for constructive interference when an X-ray beam interacts with a periodic structure [13]:

$$
n \lambda=2 d \sin \theta
$$

This means that for an X-ray beam incident with angle $\theta$ on a crystal with a lattice spacing of $d$, the resulting wavelengths that are diffracted from the crystal are integer multiples of $\lambda$.

Perfect crystals are suitable for use with synchrotron radiation where the flux of the white beam is high enough for the intensity of the single reflected energies to be sufficient for realistic acquisition times.

For use with a conventional X-ray source a mosaic crystal is preferable. A mosaic crystal is made up of smaller crystallites, each with its own orientation within the crystal, but distributed with a certain angular spread (mosaic spread) around a certain orientation to the surface. A quasi-monochromatic beam with a Gaussian distribution of energies around the monochromatic peak is produced (figure 2). This provides a good compromise between flux and energy spread. 


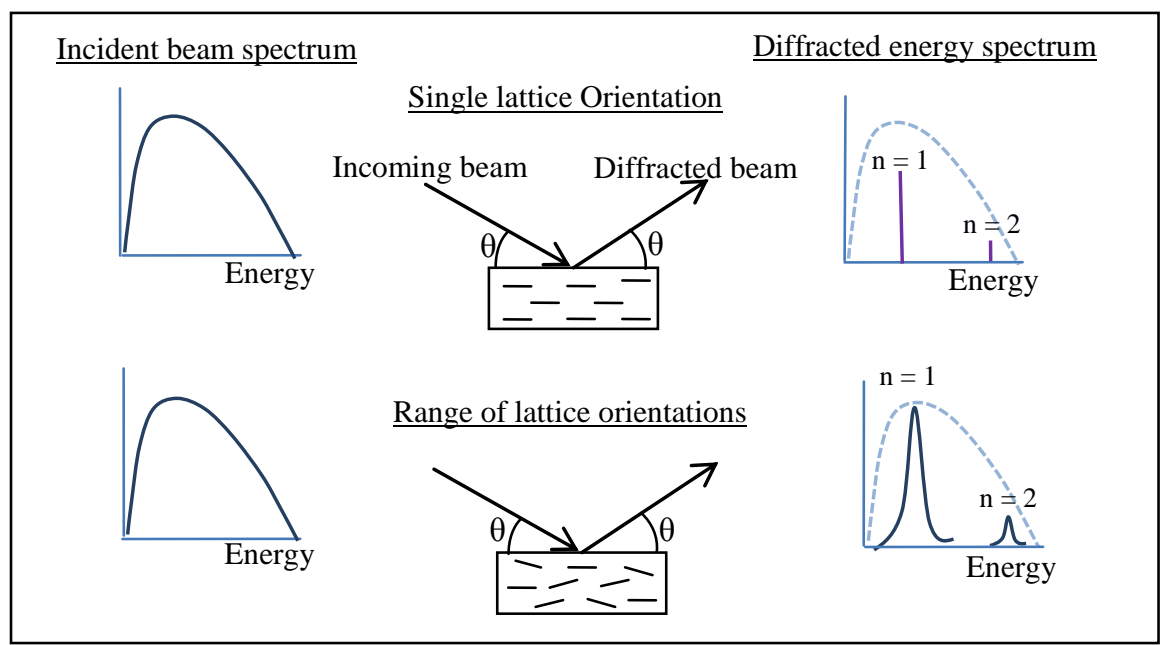

Figure 2. A diagram depicting the spectrum before and after diffraction with a crystal with a single lattice orientation (top) and with a range of lattice orientations (bottom). The diffracted spectrum shows the first two harmonics for a given incidence angle ( $n=1$ and $n=2)$.

b. Crystal characterisation

A characterisation of the beam was carried out by imaging with the setup described but without a test object. The beam was characterised at a range of angles between $2.5^{\circ}$ and $6.0^{\circ}$, corresponding to $35 \mathrm{keV}$ and $15 \mathrm{keV}$.

\section{c. low contrast test object}

The quantitative test object, shown in figure 3, is made from a Perspex block $10 \mathrm{~cm} \times 10 \mathrm{~cm} \times 0.5 \mathrm{~cm}$ in size with 6 low contrast cylindrical inserts; two different sizes $(3 \mathrm{~mm}$ and $5 \mathrm{~mm})$ and three different materials (nylon, water and wax). The test object includes several additional Perspex blocks of the same width and height but with varying thicknesses so different breast thicknesses can be simulated.

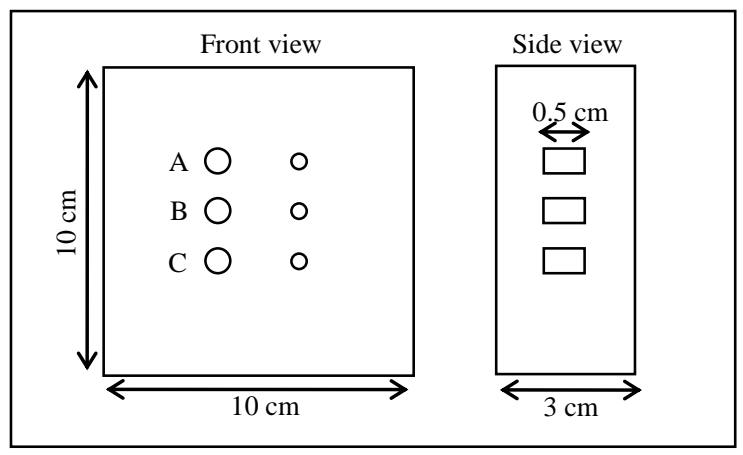

Figure 3, A front and side view diagram of the low contrast test object. A is the Nylon insert, $B$ is Air and $\mathrm{C}$ is the Wax insert. Each material has two inserts, one $5 \mathrm{~mm}$ and the other $3 \mathrm{~mm}$ in diameter. The test object is 10 cm x $10 \mathrm{~cm} \times 0.5 \mathrm{~cm}$. 


\section{d. Pixellated spectroscopic detector}

The sensor is a $20 \mathrm{~mm} \times 20 \mathrm{~mm}$ x $1 \mathrm{~mm}$ CdTe and the detector array has a $250 \mu \mathrm{m}$ pitch [14]. The detector consists of an $80 \times 80$ array of pixels, each producing an energy spectrum of the detected X-rays. The sensor is bump bonded to the $80 \times 80$ array of channels of the HEXITEC ASIC. Each channel has an identical set of electronics associated with it [15].

The detector assembly contains a Peltier cooling system to enable sub-room temperature operation.

\subsection{Image Acquisition and processing}

The low contrast test object was imaged using a "step and shoot" method. An incident angle of $2.9^{\circ}$ was used which corresponds to a peak monochromatic energy of $31 \mathrm{keV}$. The beam used is laminar so strips of images with a Gaussian vertical profile were acquired. The height of each strip is dependent on the height of the monochromatic beam, which changes depending of the angle of the crystal. Each strip also includes an overlap on each side of the strip. This region is averaged with the overlap of neighbouring strips in order to remove any artefacts at the joints between the images.

The final images were then obtained using a MATLAB routine that combines the strips and sets the average count for the designated overlap region. A flat-field correction is carried out to remove any detector related artefacts. At this point the image can be windowed over a user specified energy range to remove the scattered component.

For comparison, an image of the test object was acquired using a Hammamatsu L6732-01 tungsten anode X-ray source with $3 \mathrm{~mm}$ of Aluminium as a filter, producing a an average energy of $31 \mathrm{keV}$ [16]. The image was taken on a Hammamastsu C7942 conventional flat panel detector.

\subsection{Contrast and signal to noise ratio (SNR)}

The contrast is a parameter that can be measured from an X-ray image in order to quantify the quality of the image. Contrast is defined as the ability to distinguish a detail from the surrounding background [2]. Contrast is given by equation 2 where $M_{\text {in }}$ and $M_{\text {out }}$ are the average signal intensities inside and outside of the detail [2].

$$
\text { contrast }=\frac{\left|M_{\text {out }}-M_{\text {in }}\right|}{\operatorname{MAX}\left(M_{\text {out }}, M_{\text {in }}\right)}
$$


The signal to noise ratio is given by the ratio between the difference in signal from the detail to the background and the standard deviation in the background signal, given by equation (3) where $A$ is the area of the detail and $\sigma_{\text {out }}$ is the standard deviation of $M_{\text {out. }}$

$$
S N R=\frac{\sqrt{A}\left|M_{\text {out }}-M_{\text {in }}\right|}{\sigma_{\text {out }}}
$$

The image quality factor (IQF) is a measure of the image quality with respect to the dose, given by equation (4) where $D$ is the dose [17].

$$
I Q F=\frac{S N R}{\sqrt{D}}
$$

\section{Results}

\subsection{Crystal Characterisation}

Displayed in figure 4 is the data for the $3.2^{\circ}$ and $5.0^{\circ}$ incident angles, corresponding to $28 \mathrm{keV}$ and 18 $\mathrm{keV}$. The full width half maximum (FWHM) for the $28 \mathrm{keV}$ peak is $2.9 \mathrm{keV}$ and the FWHM for the 18 $\mathrm{keV}$ peak is $1.2 \mathrm{keV}$. The data for the characterisation shows that the smaller $3.2^{\circ}$ angle produces $50 \%$ more spread in the beam that the $5.0^{\circ}$ angle. This is due to the smaller angle being more grazing than the larger angles which results in a greater angular spread of the beam across the crystal, producing a broader, less intense peak.

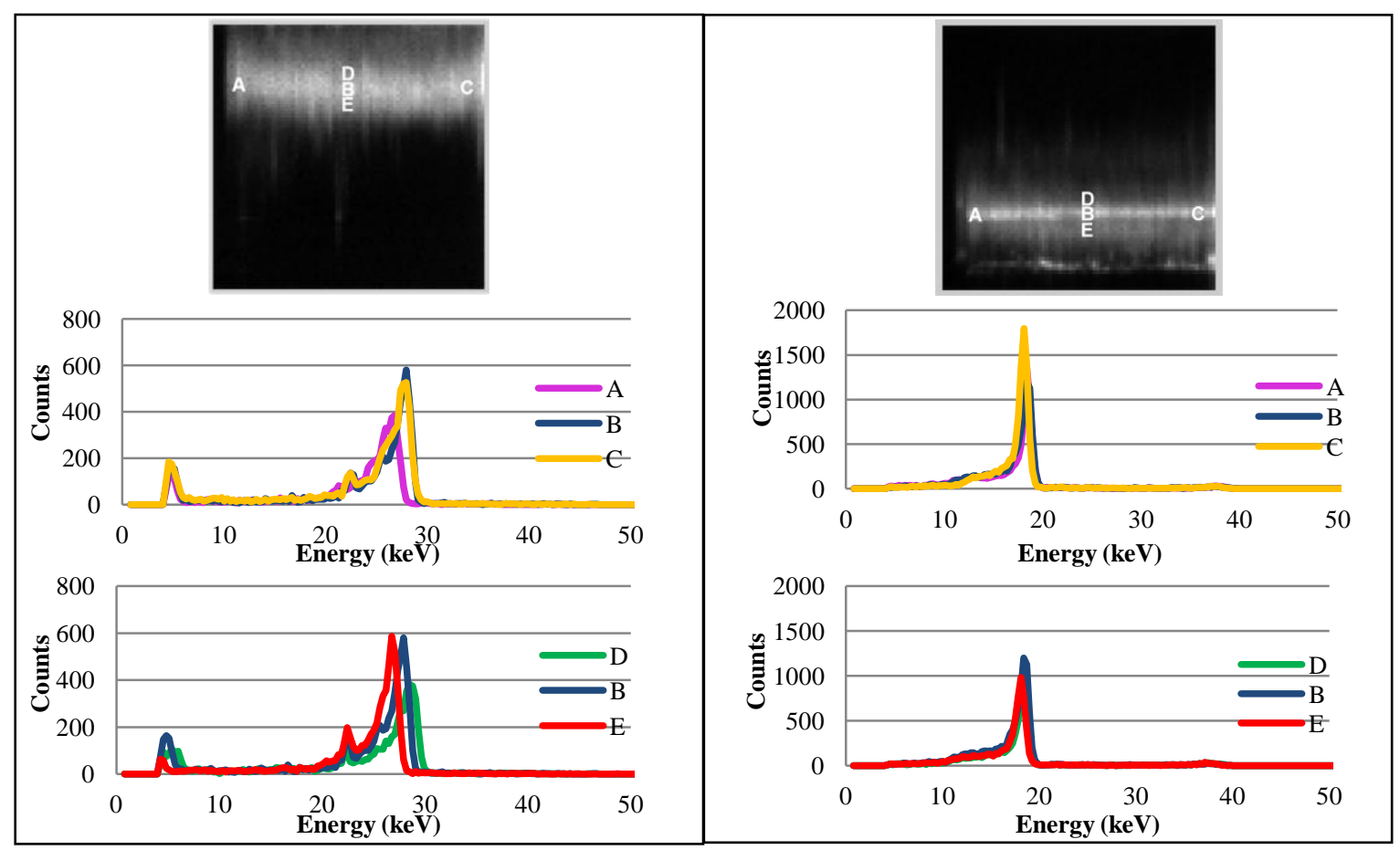

Figure 4, showing beam shape for the $3.2^{\circ}$ (left) and $5.0^{\circ}$ (right) crystal angle and graphs showing the spectral change in the vertical and horizontal axis. Points $A$ to $E$ identify the position where each spectrum was 
acquired. The rise in counts at about $5 \mathrm{keV}$ on the $3.2^{\circ}$ is the end of the low energy noise tail not completely removed from the spectrum.

\subsection{Imaging of low contrast test object}

A monochromatic image of the test object was acquired with an angle of $2.9^{\circ}$ which corresponds to an energy peak of $31 \mathrm{keV}$. Each strip of image was $2 \mathrm{~mm}$ in height with corresponds to 8 pixels on the detector with a further 2 pixels of overlap on either side of the strip.

Figure 5 shows the summed spectrum of the combined image. The monochromatic peak is identified as position M. Positions 1, 2 and 3 show the regions that the spectrum was summed over for the two conditions: the spectral region between 1 and 3 corresponds to the image with scatter and the region between 2 and 3 corresponds to the image without scatter. The energies of each of these positions is indicated in table 1.

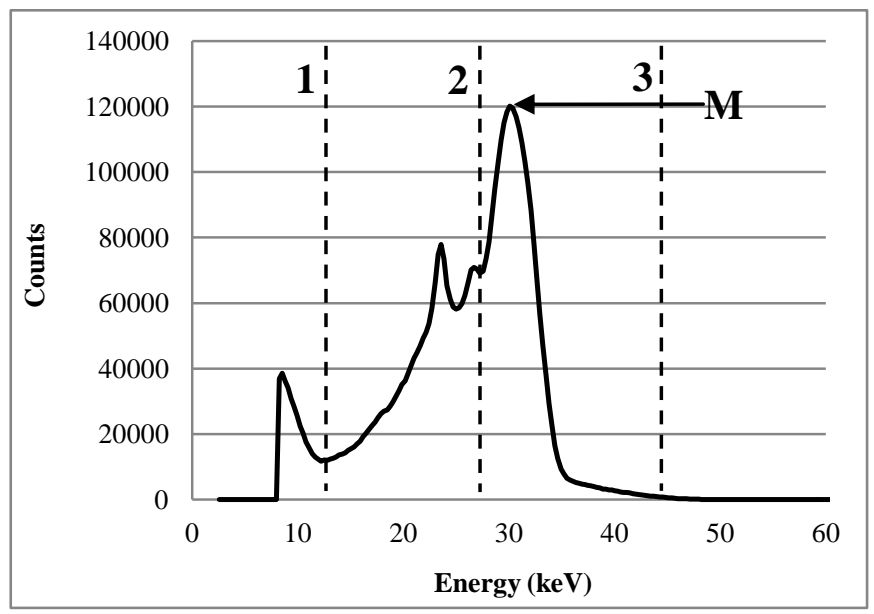

Figure 5, summed spectra for the combined image with the band including scatter (between positions 1 and 3) and the scatter-free band (between positions 2 and 3) indicated. The monochromatic peak is also shown (M). The peak at $23 \mathrm{keV}$ is a characteristic line of Cadmium [18], resulting from characteristic X-rays detected in a different pixel to the one they have been generated in. The rise in counts at about $8 \mathrm{keV}$ is the end of the low energy noise tail not completely removed from the spectrum.

The image was processed with the low energy scatter region included and excluded from the data and contrast measurements were made in each case. Figure 6 shows the final reconstructed image with the three low contrast regions identified. 


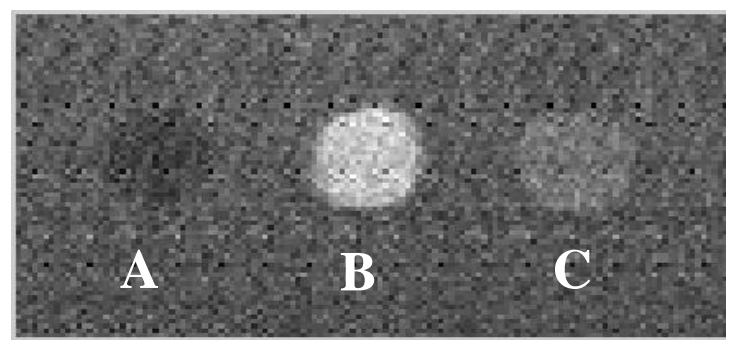

Figure 6. Final reconstructed image, before scatter correction, with the three low contrast details (Nylon (A), Air (B) and Wax (C)).

\begin{tabular}{|c|c|}
\hline Position 1 & $12.5 \mathrm{keV}$ \\
\hline Position 2 & $27.0 \mathrm{keV}$ \\
\hline Position 3 & $47.0 \mathrm{keV}$ \\
\hline Position M & $31.0 \mathrm{keV}$ \\
\hline
\end{tabular}

Table 1. Energy values for each position identified in figure 5.

The contrast for each detail was measured with the scatter component included and without the scatter component (table 2) and the results compared. The results indicate a consistent rise in the contrast when the scatter is removed from the image for the three different details.

\begin{tabular}{|c|c|c|}
\hline Detail & Contrast with low energy component & Contrast without low energy component \\
\hline Nylon (A) & $0.023 \pm 0.001$ & $0.025 \pm 0.001$ \\
\hline Air (B) & $0.124 \pm 0.001$ & $0.132 \pm 0.001$ \\
\hline Wax (C) & $0.037 \pm 0.002$ & $0.040 \pm 0.002$ \\
\hline
\end{tabular}

Table 2. The contrast values with standard errors for each detail with and without the low energy scatter component included.

The low contrast test object was also imaged using a conventional flat panel detector; the image for this is shown in figure 7. The details appear much fainter than the details for the scatter free image. The Image quality factor (equation 4) was calculated for each detail in both images and the values are shown in table 3. 


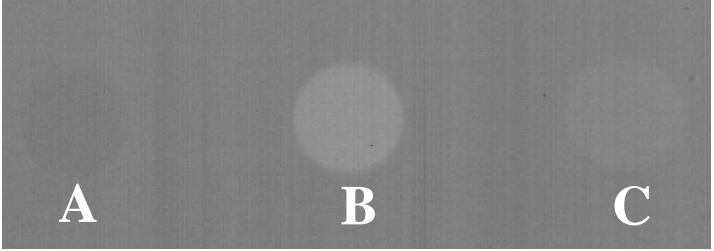

\begin{tabular}{|l|l|}
\hline Nylon (A) & $\mathbf{0 . 0 2 0} \pm \mathbf{0 . 0 0 1}$ \\
\hline Air (B) & $\mathbf{0 . 1 2 0} \pm \mathbf{0 . 0 0 1}$ \\
\hline Wax (C) & $\mathbf{0 . 0 3 4} \pm \mathbf{0 . 0 0 1}$ \\
\hline
\end{tabular}

Figure 7, shows the image of the low contrast test object taken with a conventional flat panel detector the three low contrast details (Nylon (A), Air (B) and Wax (C)) The contrast values for each detail are shown.

\begin{tabular}{|c|c|c|}
\hline Detail & $\begin{array}{c}\text { Image quality factor for HEXITEC } \\
\text { image }\left(\mu \mathrm{Gy}^{-1}\right)\end{array}$ & $\begin{array}{c}\text { Image quality factor for the conventional } \\
\text { flat panel image }\left(\mu \mathrm{Gy}^{-1}\right)\end{array}$ \\
\hline Nylon (A) & $3.5 \pm 0.5$ & $0.76 \pm 0.07$ \\
\hline Air (B) & $16.0 \pm 1.0$ & $2.9 \pm 0.2$ \\
\hline Wax (C) & $5.2 \pm 0.6$ & $0.92 \pm 0.08$ \\
\hline
\end{tabular}

Table 3, showing the contrast values with standard errors for each detail with and without the low energy scatter

\section{Conclusions}

Results from a setup for producing scatter free images with a monochromatic X-ray beam and a pixellated spectroscopic detector were presented. It has been shown that by using a pixellated spectroscopic detector with a monochromatic beam, the spectrum can be windowed around the monochromatic peak; removing the scattered component from the image. Tests using a low contrast test object showed a consistent increase in the contrast of the details.

Previous work has shown the feasibility of using monochromatic X-rays in mammography due to the removal of low-energy components and the tuneability of the energy according to the breast thickness being imaged [6],[7].

This work has shown the additional benefit resulting from coupling the monochromatic beam with a pixellated spectroscopic detector in order to remove the compton-scattered component of radiation detected and hence improve the image quality of details.

Future work will involve the investigation of different monochromatic energies on the contrast of the details in the test object. Different thicknesses of Perspex will also be investigated in order to change the scatter/primary ratio. 


\section{References}

[1] S.Webb, The physics of medical imaging, Bristol ; Philadelphia : Hilger 2012

[2] J T. Bushberg et al, The essential physics of medical imaging, Lippincott Williams \& Wilkins 2012.

[3] R L Van Metter et al, Handbook of Medical Imaging, Volume 1. Physics and Psychophysics, SPIE press 2000 .

[4] C M Ronckers et al, Radiation and breast cancer: a review of current evidence, Breast Cancer Res (2005) 7:21-32

[5] P. Sprawls, The physical principles of medical imaging, Aspen Publishers, 1993

[6] E. Burattini et al, X-ray mammography with synchrotron radiation, Review of Scientific Instruments (1992) 63638

[7] M. Gambaccini et al, Narrow energy band x-rays via mosaic crystal for mammography application, Nuclear Instruments and Methods in Physics Research A (1995) 365 248-254

[8] S D. Christe et al, The High Energy Replicated Optics to Explore the Sun Mission: A Hard X-Ray Balloon-Borne Telescope, Proc. of SPIE Vol. (2013) $8862886206-$

[9] C. Christodoulou et al, Multivariate analysis of pixelated diffraction data, (2011) JINST 6 C12027

[10] J W. Scuffham et al, Evaluation of a new small-pixel CdTe spectroscopic detector in dual-tracer SPECT brain imaging, (2012) IEEE Nuclear Science Symposium and Medical Imaging Conference Record (NSS/MIC)

[11] S. Pani et al, K-edge subtraction imaging using a pixellated energy-resolving detector, Proc. of SPIE (2011) $796179614 \mathrm{C}-1$

[12] Highly Ordered Pyrolytic Graphite Available at: http://www.2spi.com/catalog/new/hopgsub.php Accessed on 21st November 2013

[13]_S N. Ahmed, Physics and Engineering of radiation detection, San Diego; London: Academic Press 2007

[14] M C.Veale et al, X-ray microbeam characterisation of a small pixel spectroscopic CdTe detector, (2012) JINST 7 P07017.

[15] M D. Wilson et al, Multiple Module Pixellated CdTe Spectroscopic X-Ray Detector, IEEE

Transactions on nuclear science (2013) 60, 21197 - 1200 
[16] K. Cranley, 1997 Catalogue of diagnostic x-ray spectra and other data IPEM Report 78 (York: IPEM), on CD-ROM

[17] H. Aichinger et al. Image quality and dose in mammography. Electromedica (1994) 62 7-11

[18] NIST X-ray transition energies database. Available at:

http://www.nist.gov/pml/data/xraytrans/index.cfm Accessed on 30th October 2014 